\title{
Application of flow cytometry and microscopy methods in erythrophagocytosis measurement
}

\author{
ANNA STACHURSKA ${ }^{l}$, KATARZYNA GMEREK ${ }^{1,2}$, JADWIGA FABIJAŃSKA-MITEK ${ }^{1}$ \\ ${ }^{1}$ Department of Immunohaematology, Medical Centre of Postgraduate Education, Warsaw, Poland \\ 2Regional Centre of Blood Donation and Blood Therapy, Warsaw, Poland
}

\begin{abstract}
Background: Phagocytic cells engulf erythrocytes coated with IgG antibodies and/or C3 complement components in specific cellular response. Since interaction of antibody-coated RBCs with phagocytes is evaluated mainly by measurements of the phagocytic index using a subjective microscopy method, we have applied the flow cytometry technique for erythrophagocytosis research.

Aim of the study: To evaluate and compare cytometric and microscopic methods in detection and quantification of cells involved in erythrophagocytosis.

Material and methods: Monocytes and erythrocytes were isolated from the 52 human blood samples. RhD positive donor's RBCs were opsonized by specific human IgG anti-D diagnostic serum. Flow cytometry measurements of $\mathrm{CD} 14^{+}$monocytes and $\mathrm{CD} 235^{+}$erythrocytes incubated at $37^{\circ} \mathrm{C}$ or $4^{\circ} \mathrm{C}$ were performed using FACSCantoII cytometer and FACSDiva Software. Quantification of the erythrophagocytosis process was performed using cells fixed on a microscopic slide, and dynamics of this process was carried out with the use of Nikon Diaphot 300 optical microscope and Nis Elements Software.

Results: Statistical analysis of the results obtained by flow cytometry showed a lower coefficient of variation, and thus a more accurate measurement compared with the microscopic method. The flow cytometry procedure makes it possible to distinguish fully-ingested erythrocytes from these attached to the phagocyte, and hence to obtain more reliable results.

Conclusions: Flow cytometry can be an alternative technique in detection and evaluation of red blood cells phagocytosis since it is relatively rapid, more sensitive and objective than microscopy.
\end{abstract}

Key words: erythrophagocytosis, RBCs opsonization, CD235a labelling, CD14 labelling.

(Centr Eur J Immunol 2013; 38 (2): 208-213)

\section{Introduction}

Erythrophagocytosis multi-step process is the removal of senescent, damaged or abnormal red blood cells by the phagocytic cells through specific interaction of both types of cells. Despite the general knowledge concerning red blood cells destruction, the exact course of this process as well as the physiological mechanism of recognition and removal of human erythrocytes have not yet been fully explained [1]. It is known that erythrocytes may be destroyed by phagocytic cells which engulf erythrocytes coated with IgG antibodies and/or C3 complement components in specific cellular response. Immunophagocytosis is activated by the recognition of Fc antibody fragments via specific Fc $\gamma \mathrm{R}$ receptors or $\mathrm{C} 3$ complement components via $\mathrm{CR}$ receptors, present in the membrane of phagocytic cells, especially spleen and liver macrophages [2]. It is generally assumed that this interaction leads to erythrocytes destruction in two ways: 1) complete phagocytosis by phagocytic cell, 2) partial internalisation, resulting in the formation of spherocytes and microspherocytes.

In few laboratories worldwide, RBCs destruction phenomenon is used in predicting of the clinical significance of RBCs antibodies in blood recipients or patients with autoimmune haemolytic anaemia. In vitro cell assays are based on cell antibody sensitization and incubation with monocytes, which are precursors of macrophages. Phagocytosis of antibody-coated erythrocytes can be measured by microscopic monocyte monolayer assay (MMA) or the oxygen radicals measurement by chemiluminometer (CL)

Correspondence: Anna Stachurska, Department of Immunohaematology, Medical Centre of Postgraduate Education, Warsaw, Poland 
[3-5]. In addition to the above methods, it appears that the cytometric technique may also be used in erythrophagocytosis research. Flow cytometry allows for precise evaluation of the qualitative and quantitative analysis of individual cells with simultaneous analysis of large cell quantities. This method is widely used in studies of white blood cells, particularly in immunophenotypic diagnosis of lymphoproliferative disorders and immune diagnosis of deficiencies, but rarely in immunohaematological assessment of red blood cells. Therefore, the aim of this study was to evaluate and compare cytometric and microscopic methods for detection and quantification of phagocytosis. We used an antibody-dependent process, which is better known than others and is sometimes assessed in clinical trials.

\section{Material and methods}

\section{Cell isolation and erythrocyte opsonization}

An experimental system was composed of mononuclear cells and erythrocytes which were isolated from the peripheral blood of 52 healthy human donors. Written informed consent was obtained from legal representatives of all participants. The study was approved by the appropriate ethics committee. Samples of blood type $\mathrm{RhD}$ positive $(10 \mathrm{ml})$ were collected in EDTA and processed within 2 hours. Before and after isolation of cells, the sample composition was evaluated by the automated blood analyzer (Beckman Coulter, USA). Peripheral blood mononuclear cells were isolated by gradient density centrifugation on FicollHistopaque (GE Healthcare, USA) according to the $\mathrm{B} \varnothing \mathrm{yum}$ method [6]. Cell survival was tested by $0.4 \%$ Trypan Blue Solution (Hy Clone, USA). Only samples with the percentage of viable cells over $95 \%$ were used in further experiments. The mononuclear cells were suspended in RPMI1640 medium (Hy Clone, USA) supplemented with $1 \%$ PSN (penicillin, streptomycin, neomycin) and heat-inactivated $10 \%$ foetal bovine serum FBS (Hy Clone, USA).

The RhD positive RBCs were washed three times with $2 \mathrm{ml}$ of PBS, pH 6.9. After that, the $4 \%$ suspension of cells (in LISS buffer) was opsonized with IgG anti-D (polyclonal human reagent, DiaMed, Switzerland; titre 64) and verified by the anti-human IgG globulin (RCKiK, Poland).

\section{Experimental conditions}

It is known that the phagocytosis course and phagocytic activity are highly influenced by the phagocytic cells/erythrocytes ratio. Therefore, prior to the relevant experiments, a series of studies were conducted to choose the most optimal cell concentration (phagocytes/erythrocytes ratio was: $1: 100$ or $1: 1000$ ). Since the concentration with the phagocytes/erythrocytes ratio of $1: 100$ gives a higher percentage of monocytes with phagocytosed erythrocytes, we used it in further experiments.
The medium for cells incubation consisted of RPMI 1640 supplemented with Glutamax, 1\% PSN antibiotic solution and heat-inactivated foetal bovine serum (FBS), which accounted for $10 \%$ of the volume of the medium.

\section{Microscopic sequential registration}

Sequences of the images of interacting cells within $1 \mathrm{~min}$ time intervals were registered in the same microscopic field. Cells interacted for 2 hours during which phagocytosis took place. Automatic sequential microscopic image registration was carried out with the use of Nis Elements Software. Observations were examined at a stable temperature of $37^{\circ} \mathrm{C}$ $\pm 0.5^{\circ} \mathrm{C}$ with a Nikon Diaphot 300 inverted optical microscope (objective $\times 100$ ) and recorded using a CCD camera (Bischke, Germany).

\section{Microscopic phagocytosis assay}

Since the sequential registration of the dynamics of cells phagocytosis does not allow for statistical evaluation of the process, measurements at selected time interval $(1 \mathrm{~h})$ were carried out.

Monocytes were suspended in $1 \mathrm{ml}$ of RPMI medium supplemented with $10 \%$ FBS and were allowed to adhere to culture chambers slides (Nunc) for $30 \mathrm{~min}$ at $37^{\circ} \mathrm{C}$ in humidified air conditioning $5 \% \mathrm{CO}_{2}$. Non-adherent cells were removed by washing twice with $2 \mathrm{ml}$ of RPMI medium. Then, anti-D-opsonized RBCs or non-opsonized RBCs (control) were added. Incubation was carried out for $1 \mathrm{~h}$ at $37^{\circ} \mathrm{C}$ in humidified air conditioning $5 \% \mathrm{CO}_{2}$. After incubation, chamber slides were rinsed 2 times with the RPMI medium to remove the unbound erythrocytes, and subsequently the cells were fixed with methanol, dried, and stained with May-Grünwald-Giemsa reagent. Cells were observed with phase-contrast microscopy at a magnification $100 \times$.

Measurements of the number of monocytes with internalised erythrocytes and the number of erythrocytes per monocyte over a determined time interval $(1 \mathrm{~h})$ of the cells interaction made it possible to statistically estimate the investigated process.

\section{Flow cytometry}

The sample of the erythrocyte pellet $(100 \mu \mathrm{l})$ was incubated for $15 \mathrm{~min}$ at room temperature with mouse antiCD235a (glycophorin A) APC-conjugated antibodies (Becton Dickinson, USA), in a dilution of $1: 40$. The mononuclear cells suspension $(500 \mu \mathrm{l})$ was incubated with rat anti-CD14 PE-conjugated antibodies (Becton Dickinson, USA) at a final concentration of $1: 10$ for $15 \mathrm{~min}$ at room temperature. Following labelling, the two types of cells (CD235+ erythrocytes and CD14+ monocytes) were washed and co-incubated at $37^{\circ} \mathrm{C}$ with gentle stirring. One tube was incubated at $4^{\circ} \mathrm{C}$ in order to distinguish internalised erythrocytes from the adhered ones. Paired samples were prepared 
for $1 \mathrm{~h}$ : one was kept at $4^{\circ} \mathrm{C}$ as the control, and the other was incubated at $37^{\circ} \mathrm{C}$. After the incubation, cells were washed and suspended in $300 \mu 1$ of PBS. Finally, samples were studied by flow cytometry using FACSCanto II, and analysis was performed using FACSDiva Software (BD, USA). One hundred thousand events were examined in each sample. The actual percentage of monocytes with phagocytosed erythrocytes was calculated according to the formula: $\mathrm{F}=\mathrm{a}-\mathrm{b}$; $\mathrm{F}$ - monocytes with internalised erythrocytes (\%); a - mono-

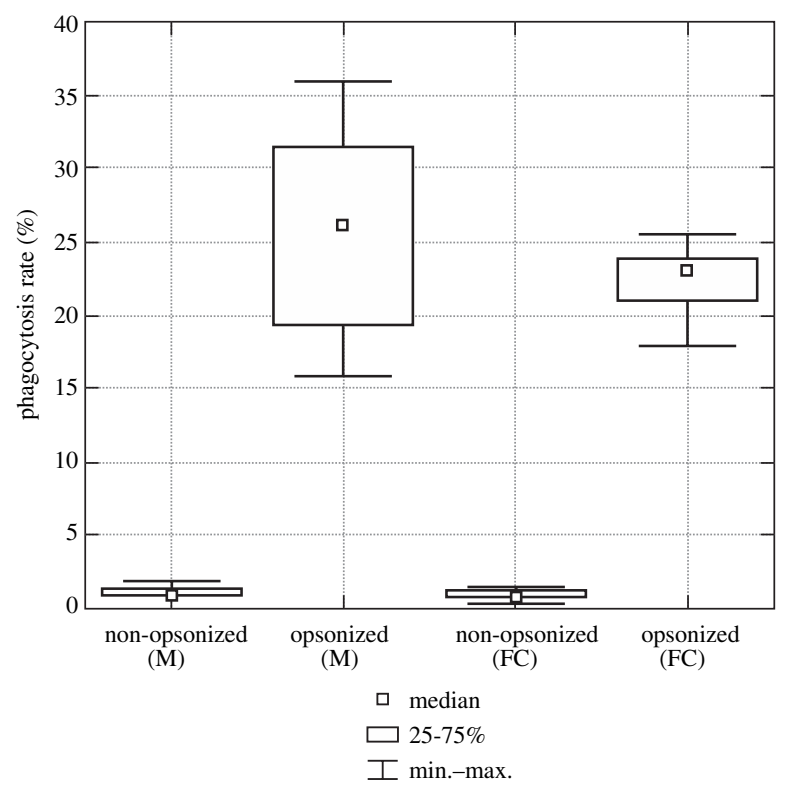

Fig. 1. Number of monocytes with internalised erythrocytes (\%); M - microscopic method, FC - flow cytometry method

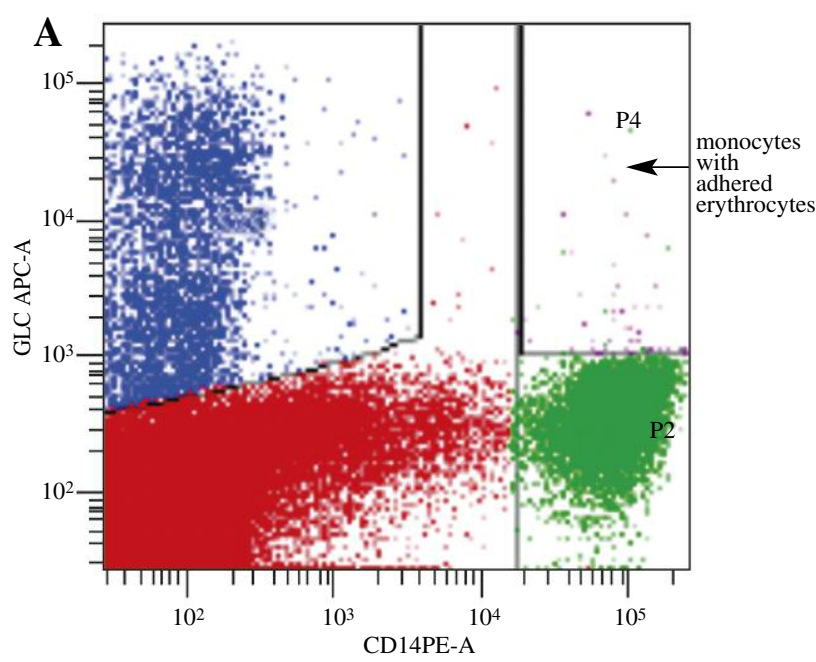

cytes with internalised and adhered erythrocytes (\%); $\mathrm{b}$ - monocytes with adhered erythrocytes (\%).

\section{Statistical analysis}

The values of monocytes with internalised erythrocytes number $(\%)$ are means of 15 measurements $(n=15)$. For statistical evaluation, a Statistica 9.0 (Statsoft) program was applied. The statistical testing of distributions of experimental results was verified by the Shapiro-Wilk test. Comparison between the mean values and the statistical significance of the differences between group means were performed using a parametric test (Student's $t$-test). The data were shown as median \pm SD values. $P<0.02$ was considered as statistically significant.

\section{Results}

Phagocytosis analysis of anti-D antibody opsonized and non-opsonized RBCs revealed that in the case of red blood cells coated with IgG anti-D, the number of monocytes with internalised erythrocytes was statistically significantly higher, as evidenced by both methods. Cytometry measurements and microscopic observations showed no significant differences in the amount of monocytes with internalised erythrocytes. Our results have shown that the percentage of monocytes with internalised erythrocytes was $26.3 \pm 6.24 \%$ using the microscopy method, and $22.2 \pm 2.45 \%$ using cytometric analysis (Fig. 1). The average percentage of monocytes with internalised erythrocytes before and after elimination of attached erythrocytes is shown in Fig. 2. It ranged from $2.3 \%$ to $3.6 \%$.

Statistical analysis of the results obtained by flow cytometry shows interquartile range lower values (3\%), and

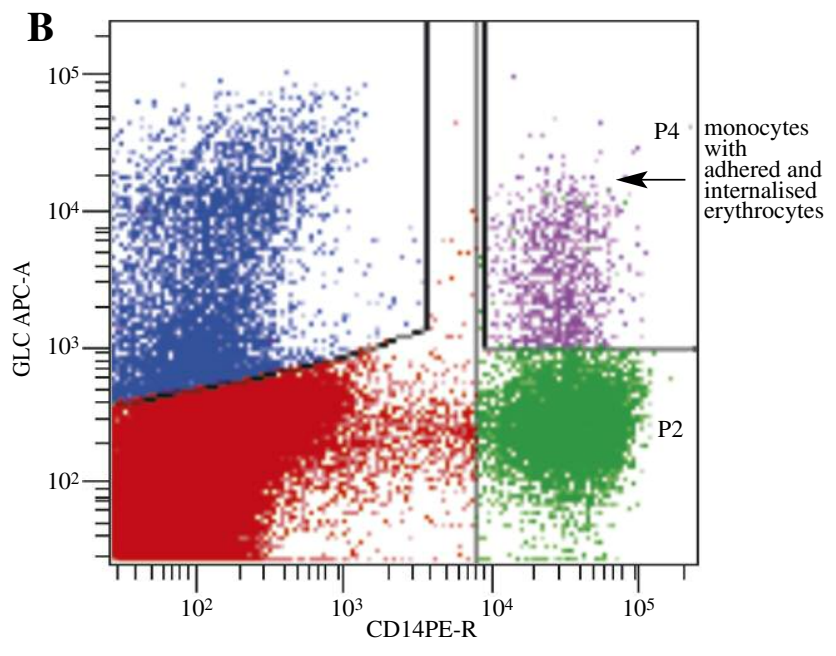

Fig. 2. Flow cytometric "dot-plot" analysis of monocytes with anti-D-opsonized erythrocytes at $4^{\circ} \mathrm{C}(\mathrm{A})$ and $37^{\circ} \mathrm{C}(\mathrm{B})$. P3 - anti-glycophorin A labelled erythrocytes; P2 - anti-CD14 labelled monocytes 
A

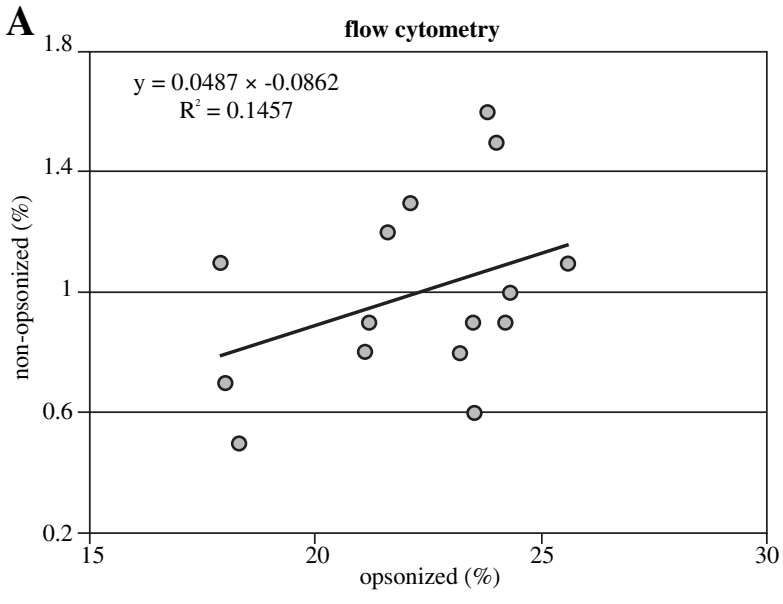

B

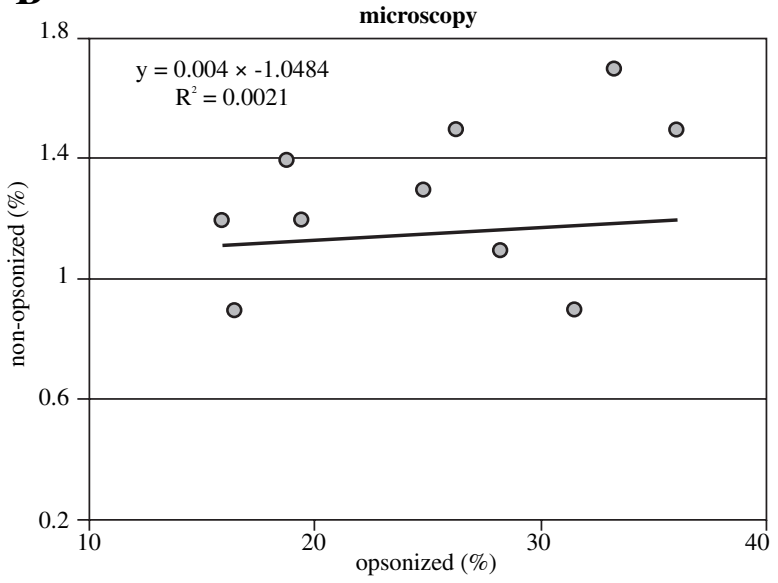

Fig. 3. Comparison of results of non-opsonized and opsonized erythrocytes $(\%)$ determined by the flow cytometric and microscopic methods
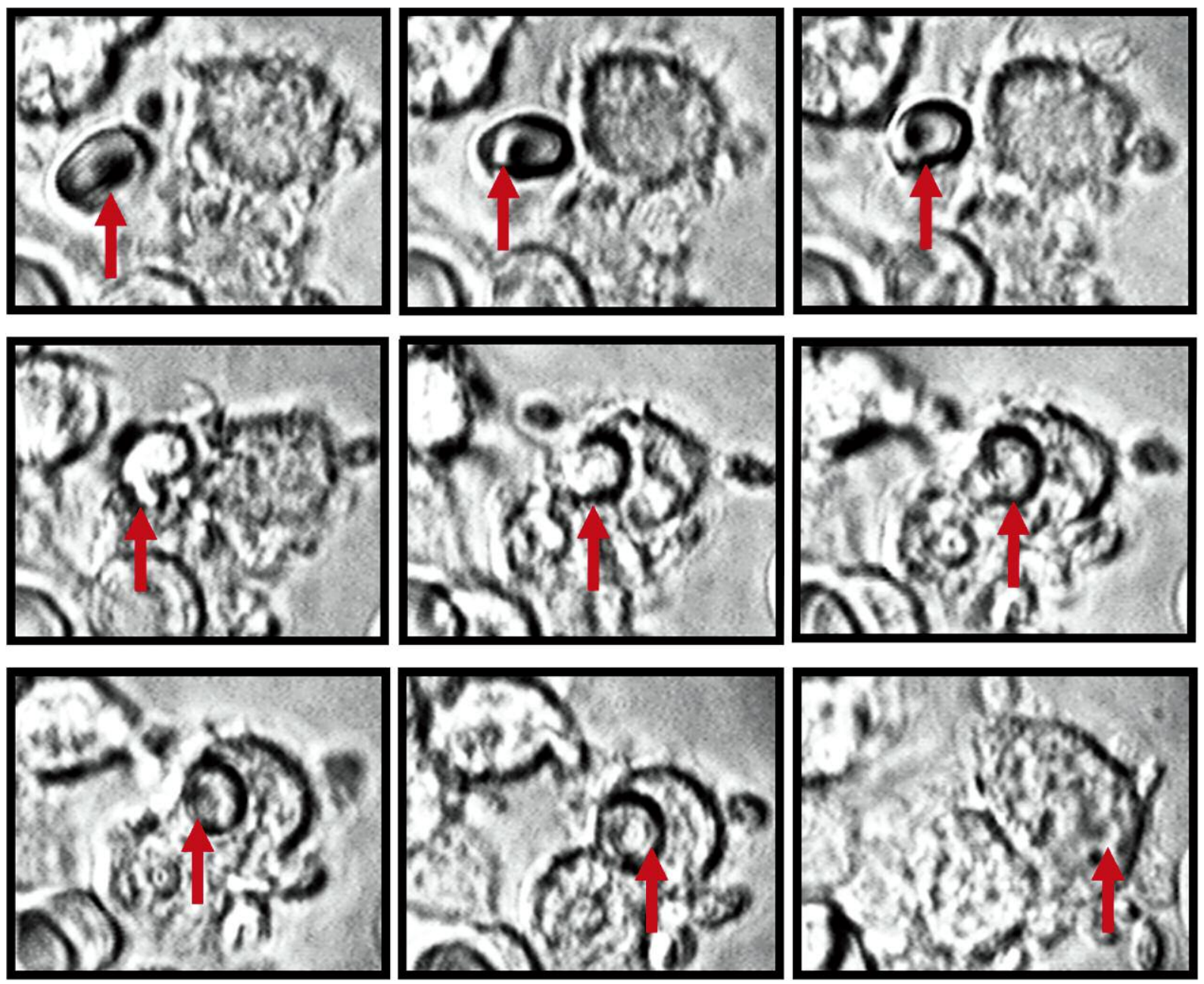

Fig. 4. Microscopic images obtained using sequential registration showing: the engulfment of erythrocyte (arrowhead) by the phagocytic cell 
thus more accurate measurements compared with the microscopic method (12\%) (Fig. 1). Cytometric values less variability in comparison to those obtained with the microscopy are shown in Fig. 3.

The analysis of microscopic images revealed that 1-3 erythrocytes were engulfed by one phagocytic cell: $60.2 \%$ phagocyted one, $30.4 \%$ - two and $9.4 \%$ - three erythrocytes. Figure 4 presents the process of phagocytosis which started 40 minutes after registration and finished after 50 minutes.

\section{Discussion}

To explain the mechanism of the phagocytosis process, most authors have used cell lines and animal cells [7-9]. However, in culture monocytes a lot of phenotypic changes occur and lose surface receptors within the first $48 \mathrm{~h}$ after isolation, and then start to differentiate into macrophages. Phenotypic heterogeneity is systematically observed in cultures of various lines of genetically identical cells even in controlled environments [10]. Additionally, some studies have shown that the structure of the cell membrane and expression changes of surface markers may affect phagocytosis of red blood cells [11-13]. Surface modulations as phospholipids externalisation (like phosphatidylserine), decreased membrane fluidity, changes of sialic acid levels and opsonins may be senescence signals recognised by phagocytic cells. However, the exact mechanism of the complex erythrophagocytosis process remains unclear and relative contribution of the above "dead signals" is not known. Therefore, it is necessary to have a good tool for evaluation of this process.

Methods of phagocytosis studies used so far are based mainly on measurements of the phagocytic index calculated based on the percentage of phagocytic cells able to engulf the red blood cells and the number of erythrocytes internalised by a single phagocytic cell $[4,14,15]$. The disadvantage of these microscopic studies of fixed cells are difficulties in distinguishing fully-ingested red blood cells from erythrocytes attached to the phagocyte. Our results indicate that a flow cytometry technique could be an alternative method in studying phagocytosis of coated red blood cells. This method is relatively rapid and sensitive, does not require large volumes of blood and allows for a more objective, reproducible results $[9,16]$. Flow cytometric assay provides an approach to obtain accurate quantitative assessment of the process by measuring the fluorescence intensity. The addition of antibodies specific of cell surface markers allows for differentiation of the respective cell populations. We used anti-CD14 antibody to identify monocytes, whereas Ig anti-glycophorin A was useful for erythrocytes separation. In addition, we can distinguish separated regions as internalised and phagocytic cells during cytometric analysis. It is worth noting that the method of cell incubation at a low temperature allows to obtain objective results and exclude adhered cells. Other investigators have used trypan blue, which has the advantage of quenching of fluorescence derived from adhered erythrocytes $[17,18]$. However, this dye can be toxic and influence the cells membrane structure, damaging surface receptors and affecting the cell interactions [19].

A commonly used fluorescence labels in cytometric and microscopic methods are $\mathrm{PKH}$, lipophilic fluorescence dyes [7-9]. However, there are some limitations concerning application of these dyes. Buffers which are used during the staining procedure have different isotonicity, what causes a significant stress on erythrocytes. After co-incubation with erythrocytes, monocytes are washed with ethanol to remove fluorescent ghosts and membrane remnants from the monocyte surface. Therefore, after this dying procedure, monocytes cannot be used in any subsequent research, due to decreased survival [20].

We do not suggest a complete abandonment of the microscopic method, but it can complement flow cytometry studies. Concomitant use of flow cytometry in addition to qualitative analysis allows for precise assessment of phagocytising monocytes and internalised erythrocytes. An application of a more objective (than the microscopic technique) cytometric method may be useful for the laboratory diagnosis of immunohaemolytic anaemias caused by allo- and autoantibodies, as well as to study other extracellular or intracellular mechanisms in the process of RBCs phagocytosis.

The authors declare no conflict of interest.

\section{References}

1. Lee SJ, Park SY, Jung MY, et al. (2011): Mechanism for phosphatidylserine-dependent erythrophagocytosis in mouse liver. Blood 117: 5215-5223.

2. Bratosin D, Mazurier J, Tissier JP (1998): Cellular and molecular mechanisms of senescent erythrocyte phagocytosis by macrophages. Biochimie 80: 175-195.

3. Hadley A, Wilkes A, Poole J, et al. (1999): A chemiluminescence test for predicting the outcome of transfusing incompatible blood. Transfus Med 9: 337-342.

4. Arndt PA, Garratty G (2004): A retrospective analysis of the value of monocyte monolayer assay results for predicting the clinical significance of blood group alloantibodies. Transfusion 44: 1273-1281.

5. La France DR, Hawthorne L, Ong M, et al. (2010): Acute hemolysis in a patient with anti-U despite a negative MMA test. Labmedicine 11: 659-661.

6. Boyum A (1983): Isolation of human blood monocytes with Nycodenz, a new non-ionic iodinated gradient medium. Scand J Immunol 17: 429-436.

7. Bratosin D, Mazurier J, Slomianny C, et al. (1997): Molecular mechanisms of erythrophagocytosis: flow cytometric quantitation of in vitro erythrocyte phagocytosis by macrophages. Cytometry 30: 269-274.

8. Bratosin D, Estaquier J, Ameisen JC, et al. (2002): Flow cytometric approach to the study of erythrophagocytosis: evidence 
for an alternative immunoglobulin-independent pathway in agammaglobulinemic mice. J Immunol Methods 265: 133-143.

9. Healey G, Veale MF, Sparrow RL (2007): A fluorometric quantitative erythrophagocytosis assay using human THP-1 monocytic cells and PKH26-labelled red blood cells. J Immunol Methods 2007; 322: 50-56.

10. Stockholm D, Benchaouir R, Picot J, et al. (2007): The origin of phenotypic heterogeneity in a clonal cell population in vitro. PLoS One 2007; 2: e394.

11. Dinkla S, Novotný VM, Joosten I, Bosman GJ (2012): Storage-induced changes in erythrocyte membrane proteins promote recognition by autoantibodies. PLoS One 2012; 7: e42250.

12. Mohandas N, Gallagher (2008): Red cell membrane: past, present, and future. Blood 10: 3939-3947.

13. Lee SJ, Park SY, Jung MB, et al. (2011): Mechanism for phosphatidylserine-dependent erythrophagocytosis in mouse liver. Blood 117: 5215-5223.

14. Fabron A, Baleotti WJr, Meloo AS (2004): Application of noninvasive phagocytic cellular assays using autologous monocytes to assess red cell alloantibodies in sickle cell patients. Transfus Apher Sci 31: 29-35.

15. Nance ST, Arndt PA (2004): Review: what to do when all RBCs are incompatible - serologic aspects. In: Immunohematology. The American National Red Cross, Philadelphia: 147-157.

16. Lechmann AK, Sørnes S, Halstensen A (2000): Phagocytosis: measurement by flow cytometry. J Immunol Methods 243: 229-242.

17. Nuutila J, Lilius EM (2005): Flow cytometric quantitative determination of ingestion by phagocytes needs the distinguishing of overlapping populations of binding and ingesting cells. Cytometry Part A 65A: 93-102.

18. Simons ER (2010): Measurement of phagocytosis and of the phagosomal environment in polymorphonuclear phagocytes by flow cytometry. Curr Protoc Cytom 9.31.

19. Chan LL, Wilkinson AR, Paradis BD (2012). Rapid image based cytometry for comparison of fluorescent viability staining methods. J Fluoresc: 1301-1311.

20. Fendel R, Mord Múller B, Kreidenweiss A, et al. (2007): New method to quantify erythrophagocytosis by autologous monocytes. Cytometry Part A 71A: 258-264. 